\title{
Protecting Sensitive Topics in Text Documents with PROTEXTOR
}

\author{
Chad Cumby \\ Accenture Technology Labs, Chicago IL 60601, USA \\ chad.m.cumby@accenture.com
}

\begin{abstract}
This is a demonstration of a system for protecting sensitive topics present in text documents. Our system works in a privacy framework where the topic is characterized as a multiclass classification problem in a generative setting. We show how our system helps a user redact a document in a business setting to obscure what company the text pertains to, and show some experimental results on redacting the topic for a standard text classification data set.
\end{abstract}

\section{Introduction}

Many companies are just starting to realize that the databases that they have released to partners or shared with the public, after cursory data "masking" exercises, can in fact be linked against other public sources to recover the sensitive information that they thought had been obscured. Luckily the machine learning and data mining communities have been very active in devising means to protect individual records from discovery by attacks such as these. $K$-anonymity [5], $L$-diversity [2], and noise based methods are effective both in identifying records where possible privacy breaches might occur, and in suppressing or generalizing fields until these records are protected.

Unfortunately, general techniques to protect sensitive information in text and semi-structured data have not been developed. Text datasets are often multiple times larger than structured databases, and the risks associated with divulging sensitive information in them are no less large. For example, when AOL released an "anonymized" set of user search queries, which were linked against external data sources to discover sensitive information about the users, the release was considered to be a major privacy breach. The challenge we address with our system is not just to identify words that are correlated with the sensitive topic (see 1]), but to optimally suppress those words within a confusion set of topics (for example Ford vs GM), to control possible inferences about the topic.

\section{PROTEXTOR System}

We define the problem of obscuring a sensitive topic in a text document $x$ in a generative multi-class classification framework. In our scenario, the trusted party possesses a corpus of documents $X$ from which $x$ is drawn, and the un-trusted 
party has some subset of $X$ minus $x$. All the documents in $X$ can be classified into a finite set $Y$ of classes, with the true class of $x$ being $y$. The goal of our redaction method is to perturb $x$ in such a way as to reduce the likelihood of $y$ and increase the likelihood of some $k$ closest of the other classes.

To approximate a model the attacker might have of $Y$, we build a Naive Bayes classifier from $X$ by creating a bag-of-words example from each document and training using the maximum likelihood method.

In our system, as shown in the screenshot below, we would then like to present to the user along with the document a suggested list of the most important words to suppress in order to make $y$ confusable with at least $k$ other classes of documents. We have experimented with several ordering metrics based on feature selection techniques from the text categorization domain, along with a new metric PosMin designed to explicitly minimize the posterior likelihood $P(y \mid x)$. We define this metric relative to the $k$ closest classes to $y$ (called $K$ ) as measured by the $K L$ divergence $K L\left(P(y) \| P\left(y^{\prime}\right)\right)$ for each $y^{\prime} \in Y$.

We'd like to minimize $\log (P(x \mid y))$. Notice that $\log (P(y \mid x))=\log (P(x \mid y))+$ $\log (P(y))-\log (P(x))=\log (P(x \mid y))+\log (P(y))-\log \left(\sum_{y^{\prime} \in Y} P\left(x \mid y^{\prime}\right) * P\left(y^{\prime}\right)\right)$. Here we re-estimate $P(x)$ relative to the set $K \cup y$ to obtain $\operatorname{Pos} \operatorname{Min}(x, y)=$ $\log (P(x \mid y))+\log (P(y))-\log \left(\sum_{y^{\prime} \in K \cup y} P\left(x \mid y^{\prime}\right) * P\left(y^{\prime}\right)\right)$. By varying $K$ for the same document, different sets of words will be suggested in order to vary the number of "guesses" necessary for an attacker to pick the correct class. For example, below is a table of the top 10 words picked by our metric for a document taken from the alt.atheism class from the 20 newsgroups dataset. In this dataset, the top 5 closest classes to alt.atheism in order are: soc.religion.christianity, talk.religion.misc, talk.politics.misc, talk.politics.mideast, \& talk.politics.guns. The list of words to confuse the document with soc.religion.christianity is very different than the one to confuse it with the whole top 5 . In particular the entire $k=1$ list does not contain the words jesus or christianity, since these words do not distinguish alt.atheism documents from soc.religion.christianity documents.

As we show in Sec.3 in a controlled evaluation, compared to the other feature selection metrics we tested, automated redacting with our new PosMin metric creates documents which are relatively unclassifiable up to $k$ guesses for classifiers trained on the unobscured documents.

Implementation. The PROTEXTOR implementation at Accenture has been created as an add-in panel for Microsoft Office 2007. In many large companies to reuse business materials from project to project it is necessary to remove client identifiable information. In order to comply with client contracts it is insufficient to simply remove the canonical name of the client. Additionally a user must remove abbreviations of the client as well as any uniquely identifying information that an attacker could use to deduce the client. So, in the first implementation we have created topic models for 450 of Accenture's clients from documents in a 350000 document corpus.

When a document is loaded, the add-in builds the word vector of the existing text and returns the most likely client classification along with the suggested words to redact to resemble the closest client $(k=1)$. The user can adjust the 


\begin{tabular}{|l|l|}
\hline $\mathbf{k}=\mathbf{1}$ & $\mathbf{k}=\mathbf{5}$ \\
\hline religion & faith \\
\hline dogma & christian \\
\hline system & dogma \\
\hline encourages & system \\
\hline toronto & encourages \\
\hline humans & beliefs \\
\hline beliefs & humans \\
\hline genocide & secular \\
\hline philosopher & philosopher \\
\hline prison & christianity \\
\hline
\end{tabular}

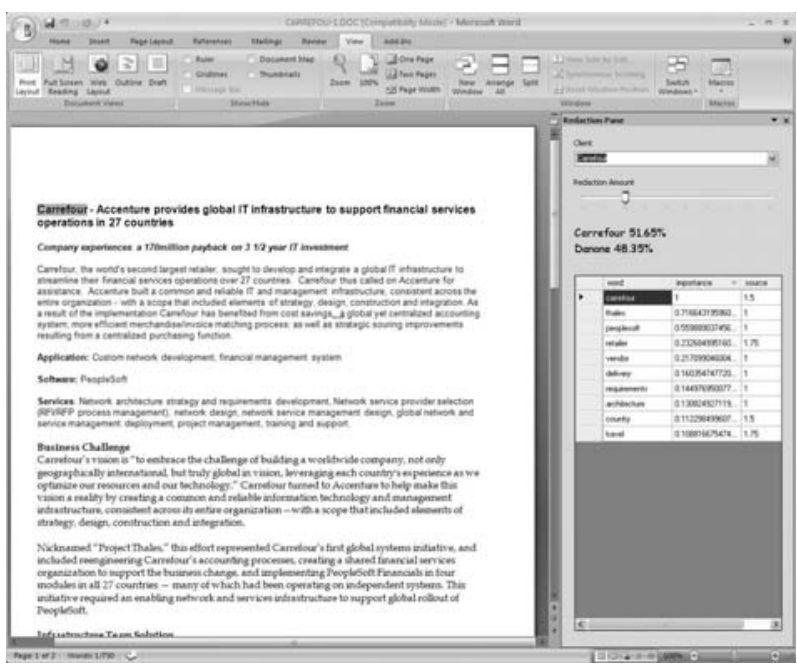

Fig. 1. (Left) Top 10 words to confuse alt.atheism doc with $k=1$ and $k=5$ other classes. (Right) Screenshot of PROTEXTOR system.

$k$ parameter via a slider to change the suggested redaction list. For example if the true client is Carrefour and the next closest client model is for Danone, the list contains the word "Carrefour" and project specific terms that distinguish the document from Danone. If the user increases the $k$ parameter to indicate that they want to obscure the document to resemble additional clients, it might suggest more general words such as "supermarket" or "france". As the user edits the text, the add-in will update the likelihoods returned for the closest client classes.

\section{Evaluation}

Here we describe a controlled experiment done in the context of the PROTEXTOR system. We set out to test what the effects of our redaction process with different parameter settings would be on learned classifiers. If our system can foil these classifiers, then an attacker scanning for sensitive information in a corpus of masked documents using them would be deterred. Also, the performance of learned classifiers seems to correlate with human performance in defeating our redaction (which we explore in further work).

The dataset used in the experiments was the Industry Sector dataset introduced in 3. It contains 6440 documents corresponding to company websites in a two level hierarchy of industry classes. We trained naive Bayes classifiers for the 12 top level classes using a standard stoplist, to serve as the class models for creating redaction word lists.

In this experiment, we created redacted sets of documents using a simple greedy redaction algorithm as follows: Given an input $k$, for each document $x$ of 


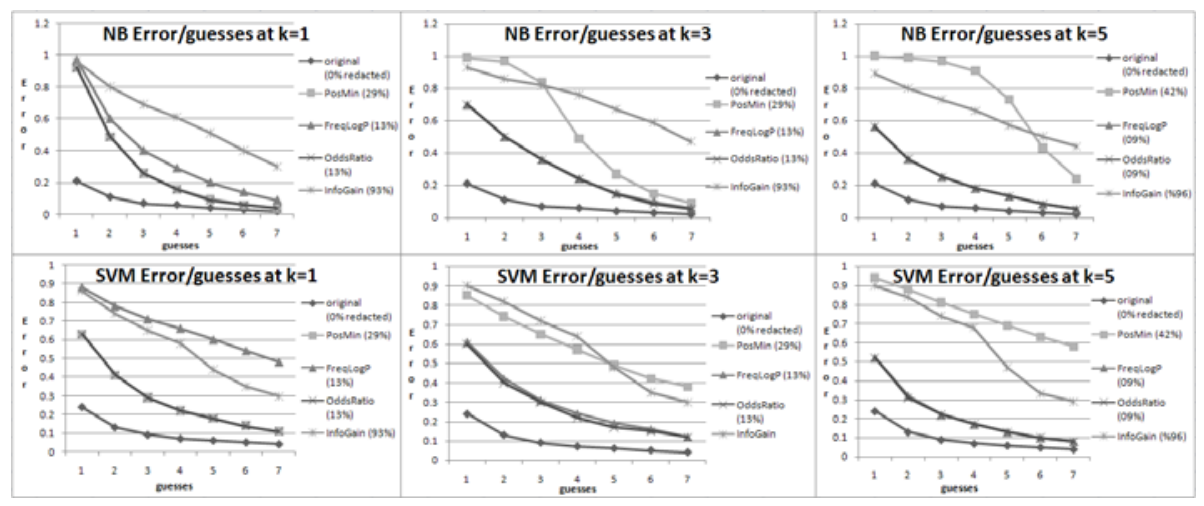

Fig. 2. All results for the Industry Sector experiment

class $y$ create an ordered list of words to redact using the four metrics below. For each list, suppress words from $x$ until the log-likelihood (LL) of $y$ is less than the LL of the $k$ closest classes. In addition to the PosMin metric as described in Sec 2, we used three standard feature selection metrics described in [4]: InfoGain, OddsRatio, and FreqLogP. Using a leave-one-out procedure we trained new naive Bayes and SVM classifiers on the corpus minus each redacted document, and tested on the redacted sets. In the results below we see the desired performance as very high error rates occur for both test classifiers up to $k$ guesses, and then a sharp drop off as the true class becomes clear. Also our PosMin metric seems to give the best performance profile at most $k$ settings, and exhibits the best tradeoff given the percentage of the document redacted.

Acknowledgements. Thanks to Yaron Rachlin for all his ideas and hard work.

\section{References}

1. Chow, R., Golle, P., Staddon, J.: Detecting privacy leaks using corpus-based association rules. In: Proceedings of KDD 2008 (2008)

2. Kifer, D., Gehrke, J.: l-diversity: Privacy beyond k-anonymity. In: Proceedings of ICDE 2006 (2006)

3. McCallum, A.K., Rosenfeld, R., Mitchell, T.M., Ng, A.Y.: Improving text classification by shrinkage in a hierarchy of classes. In: Proceedings of ICML 1998, pp. 359-367 (1998)

4. Mladenic, D.: Feature subset selection in text-learning. In: Nédellec, C., Rouveirol, C. (eds.) ECML 1998. LNCS, vol. 1398. Springer, Heidelberg (1998)

5. Sweeney, L.: Achieving k-anonymity privacy protection using generalization and suppression. International Journal on Uncertainty, Fuzziness and Knowledge-based Systems $10(2002)$ 